\title{
Search for pairs of isolated radio pulsars - components in disrupted binary systems
}

\author{
Lisa Chmyreva \\ Sternberg Astronomical Institute of $M S U$ \\ E-mail: lisa.chmyreva@mail.ru

\section{Grigory Beskin} \\ Special Astrophysical Observatory, Russian Academy of Sciences \\ E-mail: beskin@sao.ru
}

\section{Anton Biryukov}

Sternberg Astronomical Institute of MSU

E-mail: emanesai.msu.ru

\begin{abstract}
We have developed a method for analyzing the kinematic association of isolated relativistic objects - possible remnants of disrupted close binary systems. We investigate pairs of fairly young radio pulsars with known proper motions and estimated distances (dispersion measures) that are spaced no more than 2-3 kpc apart. Using a specified radial velocity distribution for these objects, we have constructed 100-300 thousand trajectories of their possible motion in the Galactic gravitational field on a time scale of several million years. The probabilities of their close encounters at epochs consistent with the age of the younger pulsar in the pair are analyzed. When these probabilities exceed considerably their reference values obtained by assuming a purely random encounter between the pulsars under consideration, we conclude that the objects may have been gravitationally bound in the past. As a result, we have detected six pulsar pairs (J0543+2329/J0528+2200, J1453-6413/J1430-6623, J2354+6155/J2321+6024, $\mathrm{J} 1915+1009 / \mathrm{J} 1909+1102$, J1832-0827/J1836-1008, and J1917+1353/J1926+1648) that are companions in disrupted binary systems with a high probability. Estimates of their kinematic ages and velocities at binary disruption and at the present epoch are provided.
\end{abstract}

25th Texas Symposium on Relativistic Astrophysics

December 6-10, 2010

Heidelberg, Germany 


\section{Introduction}

Neutron stars (NS) are among the fastest Galactic objects. Their tangential velocities determined from measured proper motions reach hundreds or even thousands of kilometers per second (see, e.g., [1] and references therein). NS probably receive such kicks during asymmetric supernova explosions [2], [3], [4], [5] or due to disruption by the explosions of the binary systems whose components they were [6], [7]. At least $50 \%$ of all stars are known to be members of binary and multiple systems [8], [9], [10], [11] and, accordingly, many of the relatively young NS that we observe as pulsars must have been formed in binary systems; their high velocities serve as additional evidence in favor of that.

NS are the final evolutionary stage of stars with initial masses in the range from 8-10M $\odot$ to $25-30 M_{\odot}$ (see, e.g., [12], [13]). Most such close binary systems (CBS) are disrupted by a supernova explosion, for which the loss of half of its mass is sufficient in the simplest case of a symmetric explosion and a circular orbit; for an asymmetric explosion, this fraction can be even smaller. Nevertheless, when one of the stars in a massive binary system explodes as a supernova at the end of its life, this does not necessarily lead to binary disruption - about $40 \%$ of the pairs withstand the explosion [13]. A CBS with a relativistic component, a NS or a black hole (BH), emerges (see, e.g., [9]). When the secondary component collapses during the subsequent evolution (the second supernova explosion occurs) and the second relativistic object is formed, the pair is generally disrupted, and the components separate: the binary as a whole is already less massive and, hence, the ejected mass (higher than that in the first case) most often exceeds the value needed for disruption.

These currently isolated NS may prove to be a convenient tool for investigating the disruption mechanism. Considering pairs of fairly close pulsars with known proper motions and distances and specifying the distribution of their space velocities, we can trace the motion of the objects in the past and determine the distance of their closest approach. If its epoch turns out to be consistent with the characteristic age $\tau_{c h}$ of one of the pulsars $\left(\tau_{c h}=P / 2 \dot{P}\right.$, where $\mathrm{P}$ is the pulsar period), then this pair can be assumed to have a common origin. The significance of this hypothesis can be estimated by comparing the probability of the encounter found with that for any two objects with arbitrary proper motions. The presence of a supernova remnant in the corresponding Galactic region that is also consistent in age with one of the pulsars can serve as an additional criterion for the validity of the identification.

Accepting the hypothesis about a common origin will allow us to analyze the second supernova explosion kinematics and the CBS disruption dynamics, as well as to ascertain the relationship between the characteristic and actual pulsar ages. We can also determine the pulsars' radial velocities and the connection between their kinematic and "internal" characteristics.

Previous attempts to discover disrupted pulsar pairs were made by various authors, including [14], [7] and [15]. An analysis of the possible association of a pair of moderately young pulsars spaced well apart for which the parallaxes and proper motions are known with a good accuracy, was undertaken by [16]. 


\section{The method}

The detailed description of the method may be found in our paper [17]. Searching for the kinematic association of two pulsars with known proper motions consists of determining the closest approaches of their reconstructed galactic trajectories in the past. Obviously, the pulsars with a common origin (i.e., those that were the components of the same binary system) were closest to each other at the time of CBS disruption. This epoch corresponds to the current age of the younger pulsar.

The law of pulsar motion $\mathbf{r}(t)$ in the Galactic gravitational potential $\varphi_{G}(\mathbf{r})$ is the solution of the equation of motion

$$
\ddot{\mathbf{r}}=-\nabla \varphi_{G}(\mathbf{r})
$$

with the initial conditions $\mathbf{r}_{0}=\mathbf{r}(t=0), \mathbf{V}_{0}=\mathbf{V}(t=0)$, where the present epoch corresponds to the initial time. Associating the pulsars, we seek for such an epoch $T$ consistent with the age of the younger pulsar (no older than its several characteristic ages) that

$$
\left|\mathbf{r}_{1}(-T)-\mathbf{r}_{2}(-T)\right|=\min \rho,
$$

where $\rho$ is the distance between the pulsars. A low value of $\min \rho$ can indicate that these objects were gravitationally bound in the past. For $\varphi_{G}(\mathbf{r})$ we used an expression that describes a threecomponent axisymmetric potential that includes the contribution from the disk-halo, bulge, and nucleus [18], [19].

The main problem in studying the kinematics of pulsars is the absence of direct measurements of their radial velocities $V_{r}$. At the same time, the source of information about the latter is the distribution function of one (any) of the three peculiar velocity components of a pulsar at the time of its birth (e.g., at the time of binary disruption). The total peculiar velocity is assumed to be isotropic relative to the LSR. Therefore, the same function can also be used for the peculiar component of the pulsar's $V_{r}$. It is constructed from the set of data on all of the investigated pulsars as a solution of the maximum likelihood problem.

Searching for the closest encounters of two pulsars is reduced to constructing the distribution of the probability $P(\rho)$ that the minimum separation between them (on a given time scale in the past) does not exceed $\rho$. First, the equation of motion was solved and sets of trajectories were simulated with various $\mathbf{r}_{0}$ and $\mathbf{V}_{0}$. The trajectories were traced into the past to a time of $(1-2) \times 10^{6}$ years back and the minimum separation $\rho_{\min }$ between them was calculated. Then the characteristic ages of the younger pulsars in the pair were compared to the epochs of closest approaches $T\left(\rho_{\min }\right)$.

Once the distribution $P(\rho)$ has been obtained, we need a criterion that would allow us to determine how significant the probability that a given pair of pulsars approached to some small distance $\rho$ in the past is. This necessary criterion consists of comparing the probabilities $P(\rho)$ and $P_{0}(\rho)$, where the latter is the probability for two randomly chosen pulsars to be at a distance no larger than $\rho$ during their motion, calculated taking into account the pulsar density in the Galaxy. If the probability $P(\rho)$ is too high for the simulated number of approaches to a distance $\rho$ to be explained only by random factors $\left(P(\rho) \gg P_{0}(\rho)\right)$, then the pair under study proves to be kinematically singled out among the others. There is reason to believe that these pulsars were once actually close to each other and to suggest that they constituted a single disrupted binary system. 


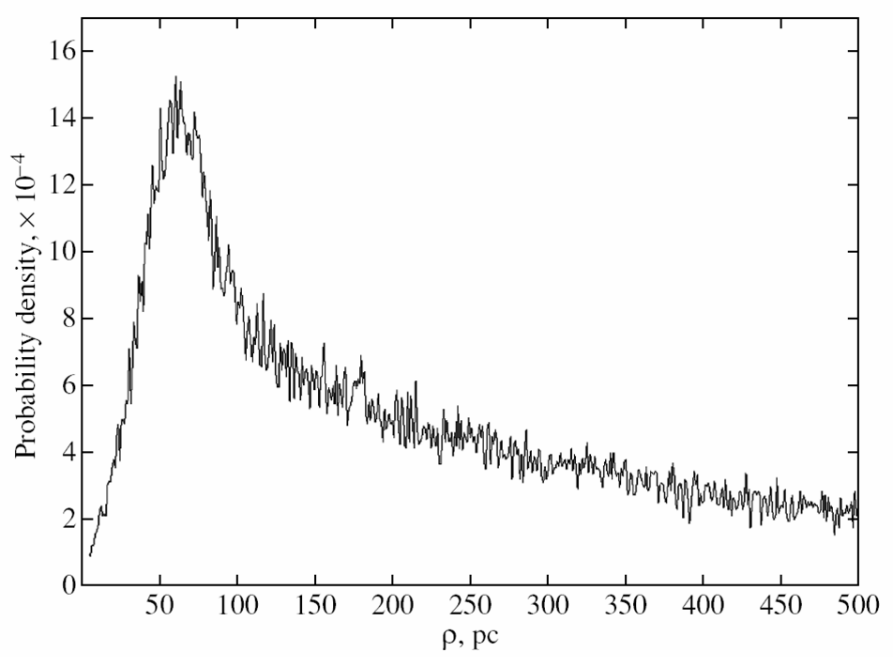

Figure 1: Probability density for the distances between J1453-6413 and J1430-6623.

\section{Results and discussion}

The possibility of a kinematic association of pulsars was considered for pairs that satisfy the following conditions: the proper motions and distances are known; the current separation does not exceed 2-3 kpc; $\tau_{c h}$ of the younger pulsar does not exceed $10^{6}$ years (the binary disruption region is determined more accurately for a small displacement of the pulsar from its current position, especially since we can be in error by a factor of several while estimating the disruption time from the characteristic age); the pulsar age difference does not exceed several million years (this follows from the typical lifetime of massive main-sequence stars).

An initial sample of $\sim 200$ isolated radio pulsars with known distances and proper motions was formed, with parameters taken mostly from the ATNF database [20] (http://www.atnf.csiro. $\mathrm{au} / \mathrm{research} / \mathrm{pulsar} / \mathrm{psrcat}$ ); we then selected pairs of objects from the sample that met the above criteria - 16 pairs in total. For each of these 16 pairs, 10000 - 15000 trajectories were simulated (per each pulsar) on a timescale of $\sim 10^{6}$ years; out of 16, we selected 7 pairs for which the probability density of the distribution of separations between the components had a distinct sharp peak at $\rho \leqslant 500$ pc (Figure 1 shows an example of such a distribution for the pair of J1453-6413 and J1430-6623). For each of the 7 pairs, a further 100000 - 300000 pairs of trajectories were simulated and the distributions $P(\rho), P_{0}(\rho)$, and $T(\rho)$ were constructed. The regions of the pairs' closest approaches were determined starting from $\rho=5$ pc. (See Tables 1 and 2.)

The best results were obtained for the pair of pulsars J0543+2329 and J0528+2200 in our simulations of 182634 trajectory pairs. The approach to $5 \mathrm{pc}$ (or closer) is realized for 81 trajectory pairs, which corresponds to the probability $P=4.435 \times 10^{4}$ at a probability of a random encounter $P_{0}$ for the same distances of less than $5 \times 10^{10}$. The difference by six orders of magnitude suggests that such a close encounter of the pulsars is not random and is indicative of their common origin. The smallest separation found between the pulsars in the past is $0.535 \mathrm{pc}$. The epoch of close encounters corresponds to $T \sim 300000$ years ago, and the characteristic age $\tau_{c h}$ of the younger pulsar $\mathrm{J} 0543+2329$ is 253000 years, which is in satisfactory agreement. If the separation between the 

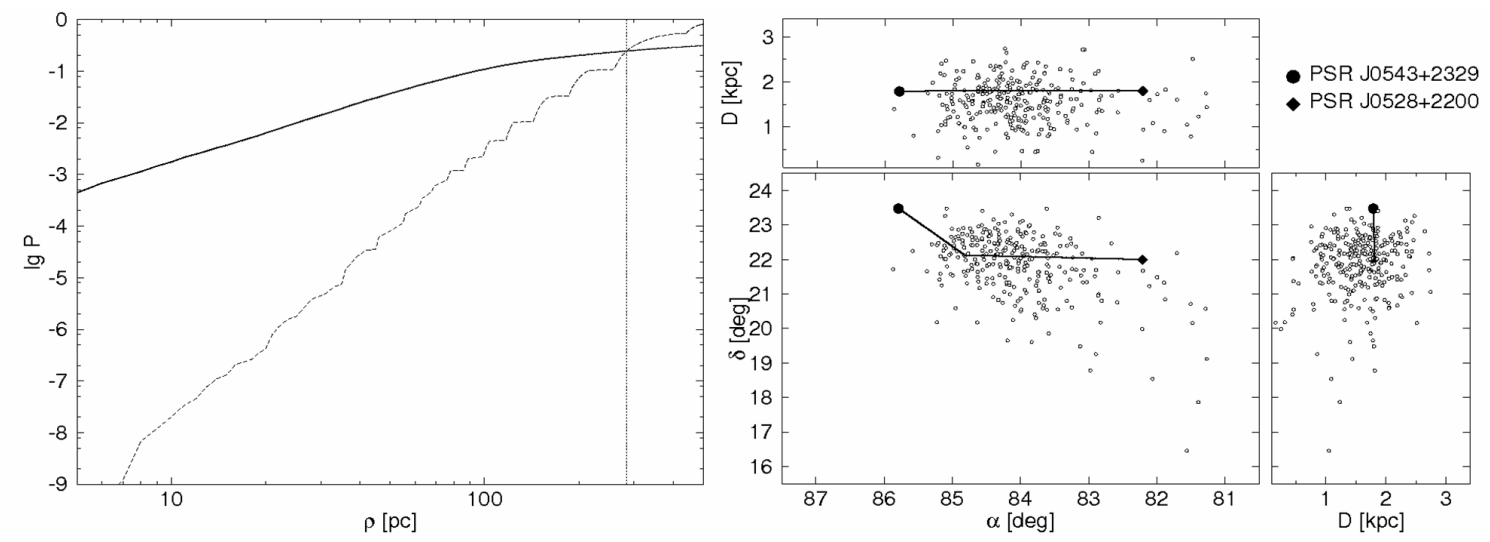

Figure 2: Pulsars J0543+2329 and J0528+2200. Left: the probability $P$ (solid curve) determined during our simulations and the probability $P_{0}$ (dashed curve) of a chance (Poissonian) detection of two (or more) pulsars in a cell of space with a size $\rho$. Right: projections of the spatial region in which the measured minimum separation between the pulsars does not exceed $\rho=10 \mathrm{pc}$. Each point on the plot is the place of closest approach of the pulsars for a specific pair of trajectories. The circle and the diamond mark the position of the younger pulsar at the present epoch and the position of the older pulsar, respectively. The lines indicate the trajectories at which the separation found between the pulsars $\rho_{\min }$ is smallest. (We omit similar figures for the other pulsar pairs.)

pulsars is assumed to be at a minimum precisely at the birth time of J0543+2329, then its actual age is close to 300000 years. Since the older pulsar J0528+2200 has a characteristic age of about $1.5 \times 10^{6}$ years, its progenitor had sufficient time before its collapse, in agreement with the evolutionary scenario for massive CBSs. The median means of the current pulsar radial velocities (for approaches to a distance smaller than $5 \mathrm{pc}$ ) are 50 and $51 \mathrm{~km} / \mathrm{s}$ for J0543+2329 and J0528+2200, respectively. The total space velocities of the pulsars relative to the LSR at the time of binary disruption (when the pulsars approached to $\leqslant 5$ pc) are 322 and 369 km/s. (See Figure 2.)

High probabilities of close approaches are also characteristic of the pair of J1453-6413 and J1430-6623. Out of the 199272 simulated trajectories, 35 pairs approached to a distance $\leqslant 5 \mathrm{pc}$, i.e., the probability $P(\rho \leqslant 5 p c)$ is $1.756 \times 10^{4}$ and the probability of a random encounter $P_{0}$ is approximately five orders of magnitude lower: $3.1 \times 10^{9}$. The minimum separation during the approach was $1.096 \mathrm{pc}$. The closest approaches occurred about 570000 years ago. The characteristic age of the younger pulsar J1453-6413 is about $10^{6}$ years; given the errors, this is a good correspondence, as in the previous case. The velocities at the binary disruption (when the pulsars approached in the past) were about $300 \mathrm{~km} / \mathrm{s}$ for J1453-6413 and $400 \mathrm{~km} / \mathrm{s}$ for J1430-6623.

Good association results were also shown by pairs J2354+6155 and J2321+6024, and J1915+1009 and $\mathrm{J} 1909+1102$.

In the pairs of J1832-0827 and J1836-1008, J1833-0827 and J1836-1008, two different secondary components can be associated with the same pulsar (J1836-1008). Here, we cannot unambiguously choose one of these associations based only on our estimates of the encounter probability. The two presumed companions, J1832-0827 and J1833-0827, are similar in characteristic age, both have high velocities (as does J1836-1008), and both are at a small distance from J1836-1008. Our simulations in both cases yielded similar results, and we can only point out that both encounters 


\begin{tabular}{c|c|c|c|c|c|c|c}
\hline \hline Pulsars & $\alpha$ & $\delta$ & $\begin{array}{c}\mathrm{D} \\
(\mathrm{kpc})\end{array}$ & $\begin{array}{c}\mu_{\alpha} \\
(\mathrm{mas} / \mathrm{yr})\end{array}$ & $\begin{array}{c}\mu_{\delta} \\
(\mathrm{mas} / \mathrm{yr})\end{array}$ & $\begin{array}{c}\tau_{c h} \\
\left(10^{3} \mathrm{yr}\right)\end{array}$ & $\begin{array}{c}\Delta \\
(\mathrm{kpc})\end{array}$ \\
\hline $\mathrm{J} 0543+2329$ & $05^{h} 43^{m} 09^{s} .660$ & $23^{o} 29^{\prime} 05^{\prime \prime} .00$ & 2.06 & $19(7)$ & $12(8)$ & 253 & 0.46 \\
$\mathrm{~J} 0528+2200$ & 052852.308 & 220001.00 & 1.61 & $-20(19)$ & $7(9)$ & 1480 & \\
\hline $\mathrm{J} 1453-6413$ & 145332.737 & -641315.59 & 2.08 & $-16(1)$ & $-21.3(8)$ & 1040 & 1.08 \\
$\mathrm{~J} 1430-6623$ & 143040.872 & -662305.04 & 1.00 & $-31(5)$ & $-21(3)$ & 4490 & \\
\hline $\mathrm{J} 2354+6155$ & 235404.724 & 615546.79 & 3.43 & $22(3)$ & $6(2)$ & 920 & 0.46 \\
$\mathrm{~J} 2321+6024$ & 232155.213 & 602430.71 & 3.03 & $-17(22)$ & $-7(19)$ & 5080 & \\
\hline $\mathrm{J} 1915+1009$ & 191529.98290 & 100943.780 & 6.27 & $4(7)$ & $-10(13)$ & 420 & 2.09 \\
$\mathrm{~J} 1909+1102$ & 190948.69380 & 110203.350 & 4.18 & $-6(4)$ & $7(8)$ & 1700 & \\
\hline $\mathrm{J} 1832-0827$ & 183237.0200 & -082703.64 & 4.85 & $-4(4)$ & $20(15)$ & 161 & 0.42 \\
$\mathrm{~J} 1836-1008$ & 183653.925 & -100808.3 & 4.46 & $18(65)$ & $12(220)$ & 756 & \\
\hline $\mathrm{J} 1833-0827$ & 183340.3000 & -082731.25 & 4.66 & $-38(26)$ & $-72(55)$ & 147 & 0.24 \\
$\mathrm{~J} 1836-1008$ & 183653.925 & -100808.3 & 4.46 & $18(65)$ & $12(220)$ & 756 & \\
\hline $\mathrm{J} 1917+1353$ & 191739.7902 & 135356.95 & 3.99 & $0(12)$ & $-6(15)$ & 428 & 1.87 \\
$\mathrm{~J} 1926+1648$ & 192645.322 & 164832.78 & 5.83 & $13(11)$ & $-14(18)$ & 511 & \\
\hline
\end{tabular}

Table 1: Parameters of the seven pairs: the pulsar coordinates, the distance to the pulsar, the proper motion, the characteristic age, and the separation between the pulsars at the present epoch.

\begin{tabular}{c|c|c|c|c|c|c}
\hline \hline Pulsars & $\begin{array}{c}\rho_{\min } \\
(\mathrm{pc})\end{array}$ & $P(5)$ & $P_{0}(5)$ & $\begin{array}{c}T(5) \\
\left(10^{3} \text { years }\right)\end{array}$ & $\begin{array}{c}V_{r}(5) \\
(\mathrm{km} / \mathrm{s})\end{array}$ & $\begin{array}{c}V(5) \\
(\mathrm{km} / \mathrm{s})\end{array}$ \\
\hline $\mathrm{J} 0543+2329$ & 0.535 & $4.435 \times 10^{-4}$ & $<5 \times 10^{-10}$ & $325 \pm 92$ & $50 \pm 131$ & $\begin{array}{c}322 \pm 85 \\
369 \pm 178\end{array}$ \\
$\mathrm{~J} 0528+2200$ & & & & & $51 \pm 257$ & 3697 \\
\hline $\mathrm{J} 1453-6413$ & 1.096 & $1.756 \times 10^{-4}$ & $3.1 \times 10^{-9}$ & $566 \pm 147$ & $-156 \pm 257$ & $321 \pm 93$ \\
$\mathrm{~J} 1430-6623$ & & & & & $319 \pm 334$ & $401 \pm 118$ \\
\hline $\mathrm{J} 2354+6155$ & 1.79 & $8.513 \times 10^{-5}$ & $1.8 \times 10^{-9}$ & $279 \pm 50$ & $-253 \pm 185$ & $609 \pm 102$ \\
$\mathrm{~J} 2321+6024$ & & & & & $-283 \pm 844$ & $930 \pm 124$ \\
\hline $\mathrm{J} 1915+1009$ & 2.646 & $4.504 \times 10^{-5}$ & $2.02 \times 10^{-8}$ & $303 \pm 64$ & $-224 \pm 306$ & $616 \pm 197$ \\
$\mathrm{~J} 1909+1102$ & & & & & $-28 \pm 59$ & $283 \pm 4$ \\
\hline $\mathrm{J} 1832-0827$ & 3.098 & $8.746 \times 10^{-6}$ & $1.23 \times 10^{-8}$ & $42 \pm 17$ & $20 \pm 17$ & $735 \pm 47$ \\
$\mathrm{~J} 1836-1008$ & & & & & $4 \pm 5$ & $2068 \pm 630$ \\
\hline $\mathrm{J} 1833-0827$ & 1.73 & $8.367 \times 10^{-6}$ & $2.5 \times 10^{-9}$ & $26 \pm 1$ & $-360 \pm 360$ & $1179 \pm 805$ \\
$\mathrm{~J} 1836-1008$ & & & & & $226 \pm 157$ & $4833 \pm 1034$ \\
\hline $\mathrm{J} 1917+1353$ & 3.399 & $9.036 \times 10^{-6}$ & $<5 \times 10^{-9}$ & $696 \pm 186$ & $491 \pm 192$ & $712 \pm 346$ \\
$\mathrm{~J} 1926+1648$ & & & & & $-117 \pm 87$ & $402 \pm 215$ \\
\hline
\end{tabular}

Table 2: The results of our simulations: the minimum separation $\rho_{\min }$ found among the simulated trajectories; the probabilities $P$ and $P_{0}$ of approach to a distance $\rho \leq 5 \mathrm{pc}$; the median epoch $T$ and the $50 \%$ confidence interval that characterizes such approaches; the median values and $50 \%$ confidence intervals of the current radial velocities $V_{r}$ for this case; the median values of the pulsar peculiar velocities $V(5)$ at the epoch of close approach and the $50 \%$ confidence interval. 
are nonrandom. However, if we compare the values of $V(5)$, we will see that these velocities are too high for the association of J1833-0827 and J1836-1008. For the pulsar J1836-1008, the velocity $\mathrm{V}(5)$ reaches almost $5000 \mathrm{~km} / \mathrm{s}$, a value higher than the velocities of even the fastest known pulsars. For the association of J1832-0827 and J1836-1008, V(5) for the same pulsar decreases to $2000 \mathrm{~km} / \mathrm{s}$, a fairly high value but not so implausible. On this basis, it seems that the association of $\mathrm{J} 1832-0827$ and $\mathrm{J} 1836-1008$ is more natural.

The last pair is $\mathrm{J} 1917+1353$ and $\mathrm{J} 1926+1648$. The probability $P$ differs from $P_{0}$ by more than three orders of magnitude, suggesting a possible association of the pulsars. Their characteristic ages are similar, 428000 and 511000 years, and we cannot say which pulsar is actually younger. The encounter epoch occurs at $T \sim 696000$ years, which may correspond to the age of one of the pulsars during the formation of which the binary was disrupted.

\section{References}

[1] G. Hobbs, et al., A statistical study of 233 pulsar proper motions, MNRAS 360, 974 (2005).

[2] I. Iben, A.V. Tutukov, On the origin of the high space velocities of radio pulsars, ApJ 456, 738 (1996).

[3] D.J. Helfand, E. Tademaru, Pulsar velocity observations - correlations, interpretations, and discussion, ApJ 216, 842 (1977).

[4] I.S. Shklovskii, Possible causes of the secular increase in pulsar periods, SvA 13, 562 (1970).

[5] R.J. Dewey, J.M. Cordes, Monte Carlo simulations of radio pulsars and their progenitors, ApJ 321, 780 (1987).

[6] A. Blaauw, On the origin of the $O$ - and B-type stars with high velocities (the "run-away" stars), and some related problems, BAN 15, 265 (1961).

[7] J.R.I. Gott, et al., Runaway stars and the pulsars near the Crab Nebula, ApJ 160, L91 (1970).

[8] A.H. Batten, On the interpretation of statistics of double stars, ARA\&A 5, 25 (1967).

[9] A.V. Zasov, K.A. Postnov, General astrophysics, Vek 2, Fryazino (2006).

[10] A. Duquennoy, M. Mayor, Multiplicity among solar-type stars in the solar neighborhood. II, A\&A 248, 485 (1991).

[11] J.L. Halbwachs, M. Mayor, et al., Multiplicity among solar-type stars. III, A\&A 397, 159 (2003).

[12] K.A. Postnov, L.R. Yungelson, The evolution of compact binary star systems, LRR 9, 6 (2006).

[13] H.A. Bethe, G.E. Brown, Evolution of binary compact objects that merge, ApJ 506, 780 (1998).

[14] G.S. Bisnovatyi-Kogan, B.V. Komberg, Pulsars and close binary systems, AZh 51, 373 (1974).

[15] G.A.E. Wright, Evidence of a disrupted pulsar binary system, Natur. 277, 363 (1979).

[16] W.H.T. Vlemmings, J.M. Cordes, et al., Separated at birth: the origin of the pulsars B2020+28 and B2021+51 in the Cygnus Superbubble, ApJ 610, 402 (2004).

[17] E.G. Chmyreva, G.M Beskin, et al., Search for pairs of isolated radio pulsars - components in disrupted binary systems, AstL 36, 116 (2010).

[18] R.G. Carlberg, K.A. Innanen, Galactic chaos and the circular velocity at the sun, AJ 94, 666 (1987).

[19] K. Kuijken, G. Gilmore, The mass distribution in the galactic disk, MNRAS 239, 571 (1989).

[20] R.N. Manchester, G.B. Hobbs, et al., The Australia Telescope National Facility pulsar catalogue, AJ 129, 1993 (2005). 